Pacific Journal of Mathematics

THE $\bar{\beta}$ TOPOLOGY FOR $W^{*}$-ALGEBRAS

Jackson Neal HenRy and Donald Curtis Taylor 


\section{THE $\bar{\beta}$ TOPOLOGY FOR $W^{*}$-ALGEBRAS}

\section{J. N. HenRy AND D. C. TAYLOR*}

Let $A$ be a $W^{*}$-algebra and $A$ * its unique predual. A new locally convex topology $\bar{\beta}$ is developed for the study of the algebra $A$. It is shown that if $A$ is a type I $W^{*}$-algebra, that is either countably decomposable, commutative, or a factor, then $\bar{\beta}$ is the Mackey topology for the dual pair $\langle A, A *\rangle$. Consequently, when $A=L^{\infty}(X, \mu)$, where $X$ is completely regular and $\mu$ is a compact regular Borel measure on $X, A_{3}^{*}=L^{1}(X, \mu)$ and $\bar{\beta}$ convergence on uniformly bounded sets is equivalent to convergence in measure.

Let $X$ be a locally compact Hausdorff space, $\beta X$ the Stone-Čech compactification of $X$, and $C(\beta X)$ the collection of all complex-valued continuous functions on $\beta X$. In 1958, R. C. Buck [2] introduced a new locally convex topology for $C(\beta X)$ that gave new insight into the intricate structure of $C(\beta X)$. This locally convex topology for $C(\beta X)$, which Buck called the strict topology, is the topology generated by the seminorms $\left\{\lambda_{f}\right\}_{f \in C_{0}(X)}$, where $\lambda_{f}(g)=\|f g\|_{\infty}$. Here, $C_{0}(X)$ denotes those functions in $C(\beta X)$ that vanish on $\beta X \backslash X$. Although Buck's approach is very useful in the study of $C(\beta X), X$ locally compact, it does not lend itself to the study of $C(\beta X), X$ completely regular, since $C_{0}(X)$ may be the $\{0\}$ subspace in this setting. In [18], F. D. Sentilles was able to overcome this possibility by introducing a new topology which, in the locally compact setting, reduces to the strict topology. Sentilles' topology, $\beta$, is defined as follows: for each $Q \subseteq \beta X \backslash X$, let $\beta_{Q}$ be the strict topology on $C(\beta X)$ determined by $C_{0}(\beta X \backslash Q)$. Then $\beta$ is defined as the inductive limit of the topologies $\beta_{Q}$ as $Q$ ranges over all compact subsets of $\beta X \backslash X$ [18]. Note that $\beta$ is determined by the collection of open sets $V, \beta X \supseteq V \supseteq X$, whose Stone-Čech compactification is $\beta X$ and is therefore not a unique topology, since it depends on the underlying subspace $X$. Using this topology, substantial progress has been made in the study of $C(\beta X), X$ completely regular, by Sentilles, Wheeler and others (see [8], [18], [24], [25]).

The purpose of this paper is to define and study a noncommutative analogue of the topology introduced by Sentilles. Noncommutative versions of Buck's topology already exist in a Banach module setting [19] and in the $C^{*}$-algebra of double centralizers $M(B)$ of the $C^{*}$ algebra $B$ [3], [20], [22]. In the double centralizer setting, $B$ is viewed as a closed two-sided ideal in $M(B)$, and the strict topology for $M(B)$ is 
generated by the seminorms $\left\{\lambda_{b}, \rho_{b}\right\}_{b \in B}$, where $\lambda_{b}(x)=\|b x\|$ and $\rho_{b}(x)=$ $\|x b\|$ for $x \in M(B)$. This topology has been very useful in the study of the $C^{*}$-algebra $M(B)$. In general it would be desirable to use this approach to study $C^{*}$-algebras $A$ with identity, that is, develop a locally convex topology for $A$ with the essential properties of the strict topology. It would be natural to try to find a closed two-sided ideal $J \subseteq A$ such that $M(J)=A$, but this in general is difficult to do. Consequently, we find it necessary to place additional restrictions on our $C^{*}$-algebra. Namely, we will require $A$ to be a $W^{*}$ algebra. Here we view a $W^{*}$-algebra as a $C^{*}$-algebra which is the dual of a unique Banach space $A_{*}[14]$. In a $W^{*}$-algebra $A$, it is known that a closed two-sided ideal $J \subseteq A$ has the property that $M(J)=A$ if and only if $J$ is essential, that is, $J^{0} \equiv\{x \in A: x J=\{0\}\}=\{0\}$ (see [22]). Since it is probable that more than one ideal with this property exists, it seems natural to apply Sentilles' method to our setting. Consequently, we define the $\bar{\beta}$ topology for a $W^{*}$-algebra $A$ as follows: for each essential closed two-sided ideal $J \subseteq A$, we define the strict topology $\beta_{J}$ for $A$ to be the locally convex topology generated by the seminorms $\left\{\lambda_{a}, \rho_{a}\right\}_{a \in J}$ as in the double centralizer setting above. We then define the $\bar{\beta}$ topology to be the inductive limit of the $\beta_{J}$ topologies [13]. The algebra $A$ under the $\bar{\beta}$ topology will be denoted by $A_{\bar{\beta}}$. If $A$ is topologically simple, then the $\bar{\beta}$ topology is the norm topology, since $A$ is the only ideal $J \subseteq A$ such that $M(J)=A$. Note that our $\bar{\beta}$ topology is space free and unique while Sentilles' topology is generated by a subclass of these ideals and, consequently, in Sentilles' setting our topology is a weaker topology than his $\beta$ topology. The main question that we consider in this paper is the following: for a countably decomposable $W^{*}$-algebra (for example, $A *$ separable), what are necessary and sufficient conditions for the dual of $A_{\bar{\beta}}$, denoted $A_{\bar{\beta}}{ }^{*}$, to be $A_{*}$ ? We show that a sufficient condition is for $A$ to be a type I $W^{*}$-algebra and we have evidence to suggest it is a necessary condition as well. When $A_{\bar{\beta}} *$ is $A_{*}$, then $\bar{\beta}$ is the Mackey topology $\tau\left(A, A_{*}\right)$ as studied by Sakai [14], Akemann [1] and others. In the special case when $A$ is $L^{\infty}(\Omega, \mu), \bar{\beta}$ is the mixed topology of Dazord and Jourlin [4].

In $\$ 2$ we discuss hyper-Stonean spaces as related to a $W^{*}$-algebra and $\S 3$ is devoted to the study of essential ideals. The general study of the $\bar{\beta}$ topology is presented in $\S 4$ with our main results appearing in $\S 5$. The reader is referred to [5], [6], and [14] for definitions and basic concepts of $C^{*}$-algebras and $W^{*}$-algebras.

2. Hyper-Stonean topological spaces. Let $\Omega$ be a compact Hausdorff space and $C(\Omega)$ the space of all complex-valued continuous functions on $\Omega$. The space $\Omega$ is called Stonean if the 
closure of every open set is open, or equivalently, $C(\Omega)$ is a conditionally complete lattice $[9,3$ N. 6, p. 52]. Now suppose $\Omega$ is Stonean. A finite positive regular Borel measure $\mu$ on $\Omega$ is said to be normal if it satisfies the following property: if $\left\{f_{\alpha}\right\}$ is a uniformly bounded increasing directed set of positive functions in $C(\Omega)$, then l.u.b. $\int_{\Omega} f_{\alpha} d \mu=\int_{\Omega}$ l.u.b. $f_{\alpha} d \mu$. A finite complex regular Borel measure is called normal if it is a linear combination of positive normal measures. We denote by $M(\Omega)$ the finite complex regular Borel measures on $\Omega$ and by $N(\Omega)$ the closed subspace of normal measures. The Stonean space $\Omega$ is said to be hyper-Stonean if the union of the supports of the positive normal measures is dense in $\Omega$, or equivalently, $C(\Omega)$ is a $W^{*}$-algebra [14, p. 46].

Throughout this section we shall assume that $\Omega$ is a hyper-Stonean space. The results in this section are due to Dixmier [7] and we include them here for completeness.

2.1. PROPOSITION. Let $\left\{f_{\alpha}\right\}$ be an increasing net of continuous functions in $C(\Omega)$ which is bounded above. If $f$ is the lattice supremum and $f^{\prime}$ the upper envelope $\left(f^{\prime}(x)=\sup _{\alpha} f_{\alpha}(x), x \in \Omega\right)$, then $f$ and $f^{\prime}$ differ on a set of first category.

Proof. For the proof, see [7, p. 154].

2.2. Proposition. In order that the measure $\mu$ in $M(\Omega)$ be normal it is necessary and sufficient that $\mu(\Delta)=0$ for all nowhere dense Borel subsets $\Delta$ of $\Omega$.

Proof. For a proof, see [7, Proposition 1, p. 157].

2.3. PROPOSITION. Let $\mu$ be a positive normal measure on $\Omega$ and $f$ a $\mu$-measurable complex-valued function. Then there exists a continuous function $f^{\prime}$ on $\Omega$ such that $f=f^{\prime}$ almost everywhere.

Proof. For a proof, see [7, Proposition 2, p. 157].

2.4. Corollary. If the support of $\mu$ is $\Omega$, then $C(\Omega)$ is *isomorphic to $L^{\infty}(\Omega, \mu)$.

We note that by $\left[14,1.2 .6\right.$, p. 5] every *-isomorphism of $C^{*}$ algebras is an isometry.

2.5. Proposition. Let $\mu$ be a positive normal measure on $\Omega$ and $\Delta$ a $\mu$-measurable subset of $\Omega$. Then $\Delta$ coincides, except on a set of $\mu$-measure zero, with the closure $\bar{\Delta}$, with the interior $\Delta^{i}$, with the closure of $\Delta^{i}$, and with the interior of $\bar{\Delta}$. 
Proof. For a proof, see [7, Corollary, p. 158].

2.6. Corollary. The support of $\mu$ is both open and closed.

2.7. Corollary. If the support of $\mu$ is $\Omega$ and $\Delta$ is a $\mu$ measurable set such that $\mu(\Delta)=0$, then $\Delta$ is nowhere dense.

A measure space $(\Gamma, \nu)$ is said to be localizable if there exists a family $\left\{\left(\Gamma_{\alpha}, \nu_{\alpha}\right)\right\}$ of finite measure spaces such that $\Gamma=\cup \Gamma_{\alpha}, \nu=\Sigma \oplus \nu_{\alpha}$, and the family $\left\{\Gamma_{\alpha}\right\}$ is pairwise disjoint. Note that $L^{\infty}(\Gamma, \nu)=$ $\Sigma \oplus L^{\infty}\left(\Gamma_{\alpha}, \nu_{\alpha}\right)$. The measure space $(\Gamma, \nu)$ is called $W^{*}$-localizable if each $\Gamma_{\alpha}$ is a hyper-Stonean space and $\nu_{\alpha}$ is a positive normal measure on $\Gamma_{\alpha}$ with support $\Gamma_{\alpha}$.

2.8. Proposition. Let $Z$ be a commutative $W^{*}$-algebra. Then $Z$

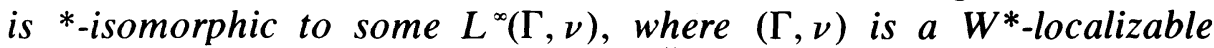
measure space. Moreover, the Stone-Čech compactification of $\Gamma$ is the spectrum of $Z$.

Proof. Since $Z$ is *-isomorphic to $C(\Omega), \Omega$ hyper-Stonean, the result follows from the proof of [7, Theorem 1, p. 169].

3. Essential ideals in $W^{*}$-algebras. Let $A$ be a $W^{*}$ algebra and $J$ a closed two-sided ideal of $A$. The ideal $J$ is called essential if $J^{0} \equiv\{x \in A: x J=\{0\}\}=\{0\}$. The essential ideals of $A$ will be denoted by $\mathscr{E}_{A}$, or $\mathscr{E}$ is $A$ is understood. We do not assume $J$ is proper.

A double centralizer of the ideal $J$ is an ordered pair $(S, T)$ of functions from $J$ to $J$ such that $x S(y)=T(x) y$ for all $x, y$ in $J$. In [3] Busby shows $S$ and $T$ are bounded linear maps with $\|S\|=\|T\|$ and the space of all double centralizers of $J$, denoted by $M(J)$, is a $C^{*}$-algebra under the natural algebraic operations and norm $\|(S, T)\|=\|S\|$. There is a natural embedding of $A$ into $M(J)$, namely, the map $x \rightarrow\left(L_{x}, R_{x}\right)$ where $L_{x}(y)=x y$ and $R_{x}(y)=y x$ for all $y \in J$. Our next result connects double centralizer algebras and essential ideals. For basic concepts and definitions of double centralizers, we refer the reader to [3], [20] and [22].

3.1. Lemma. Let $J$ be a closed two-sided ideal of the $W^{*}$-algebra A. Then the map $x \rightarrow\left(L_{x}, R_{x}\right)$ is a *-homomorphism of $A$ onto $M(J)$. Moreover, the map is $a^{*}$-isomorphism if and only if $J$ is essential. 
Proof. Let $A_{0}$ be the $W^{*}$-subalgebra of $A$ generated by $J$. It is easy to show that $J$ is essential in $A$. The conclusion follows from [22, Theorem 2.1 and Corollary 2.2, p. 478].

3.2. Proposition. Let $A$ be $a W^{*}$-algebra and $I, J$ and $K$ closed two-sided ideals of $A$. The following statements are true:

(1) If $J \subseteq K$ and $J \in \mathscr{E}$, then $K \in \mathscr{E}$.

(2) If $I, J \in \mathscr{E}$, then $I+J \in \mathscr{E}$.

(3) If $I, J \in \mathscr{E}$, then $I \cap J \in \mathscr{E}$.

Proof. The proof of (1) is trivial. It is well-known that $I+J$ is a closed two-sided ideal, so (2) follows immediately from (1). It is straightforward to show, by utilizing 3.1, that $\|x\|=\sup \{\|x y\|: y \in I \cap J$, $\|y\| \leqq 1\}$, since $I$ and $J$ are essential. Thus the map of 3.1 is an isometry and (3) follows.

The next result shows that $W^{*}$-algebras in general have an ample supply of essential ideals.

3.3. Proposition. Let $A$ be a $W^{*}$-algebra. Then $A$ can be written as follows: $A=\Sigma_{\alpha \in \pi} \oplus A_{\alpha}$, where each $W^{*}$-algebra $A_{\alpha}$ is either topologically simple or each maximal two-sided ideal of $A_{\alpha}$ is essential with respect to $A_{\alpha}$.

Proof. Let $F$ be the family of all sets $\left\{P_{\alpha}\right\}$ of central projections with the following properties: (1) $P_{\alpha} P_{\beta}=0$ for $\alpha \neq \beta$; (2) $P_{\alpha} A$ is topologically simple. It is easy to see, by using Zorn's lemma, that there is a maximal such family $\left\{P_{\alpha}\right\} . \quad$ Let $A_{\alpha}=P_{\alpha} A$ and $P=\Sigma P_{\alpha}$. It is straightforward to verify that $A=\left(\Sigma \oplus A_{\alpha}\right) \oplus(1-P) A$. Now suppose $J$ is a maximal ideal of $(1-P) A$ that is not essential. It follows that $J^{0}$ is a nonzero topologically simple two-sided ideal of $(1-P) A$ which is closed in the $\sigma\left(A, A_{*}\right)$ topology. Therefore, there is a central projection $Q$ such that $Q A=J^{0}[14,1.10 .5$, p. 25]. But this contradicts the fact that $\left\{P_{\alpha}\right\}$ was maximal. Hence our proof is complete.

It is well known that a factor contains a smallest nonzero, not necessarily proper, closed two-sided ideal [26, Remark 3, p. 61]. We will use this fact in the following proposition.

3.4. Proposition. Suppose that the $W^{*}$-algebra $A$ is a factor. Then every nonzero closed two-sided ideal of $A$ is essential.

Proof. Let $J$ be the smallest nonzero closed two-sided ideal of A. By virtue of 3.2 , we need only show $J$ is essential. If $J^{0} \neq\{0\}$, then 
$J \subseteq J^{0}$. But this is clearly a contradiction. Hence $J^{0}=\{0\}$ and our proof is complete.

Let $(\Omega, \mu)$ be a localizable measure space and $A$ a $W^{*}$-algebra with separable predual $A_{*}$. We let $L^{\infty}(\Omega, \mu, A)$ denote the Banach space of all $A$-valued essentially bounded weakly* $\mu$-locally measurable functions on $\Omega$ (see [11, 3.5, p. 72]). In [14, 1.22.13, p. 68], Sakai shows $L^{\infty}(\Omega, \mu, A)$ is a $W^{*}$-algebra under pointwise multiplication and its predual is $L^{\prime}\left(\Omega, \mu, A_{*}\right)$, where $L^{1}\left(\Omega, \mu, A_{*}\right)$ is the Banach space of all $A_{*}$-valued Bochner $\mu$-integrable functions on $\Omega$. The next lemma connects $W^{*}$-tensor products with the space $L^{\infty}(\Omega, \mu, A)$. For basic definitions and concepts of tensor products of $C^{*}$-algebras, we refer the reader to $[14,1.22, \mathrm{pp} .58-70]$. For the definition of the $s(A, A *)$ and $s^{*}\left(A, A_{*}\right)$ topologies see, $[14$, p. 20].

3.5. Lemma. Let $Z$ be a commutative $W^{*}$-algebra and $A$ a $W^{*}$-algebra with separable predual. Then $Z \bar{\otimes} A$ is ${ }^{*}$-isomorphic to $\Sigma_{\alpha \in \pi} \oplus L^{\infty}\left(\Omega_{\alpha}, \mu_{\alpha}, A\right)$, where each $\Omega_{\alpha}$ is hyper-Stonean and $\mu_{\alpha}$ is a positive normal measure with support $\Omega_{\alpha}$.

Proof. The proof follows immediately from 2.8 and $[14,1.22 .13$, p. $68]$.

3.6. LemmA. Let $Z$ be a commutative $W^{*}$-algebra and $A$ a factor with $A *$ separable. If $J$ is a closed two-sided ideal of $Z \bar{\otimes} A$ such that $J \cap\left(Z \bigotimes_{\alpha_{0}} A\right)=\{0\}$, then $J=\{0\}$.

Proof. By virtue of 3.5 we may assume $Z \bar{\otimes} A=L^{\infty}(\Omega, \mu, A)$, where $\Omega$ is hyper-Stonean and $\mu$ is a positive normal measure with support $\Omega$. Moreover, by virtue of 2.4 and [14, 1.22.3, p. 61], we may assume $Z \bigotimes_{\alpha_{0}} A=C(\Omega, A)$, where $C(\Omega, A)$ is viewed as a subalgebra of $L^{\infty}(\Omega, \mu, A)$ in the natural way. Note that it follows from 2.4 that the center of $L^{\infty}(\Omega, \mu, A)$ is $C(\Omega) \cdot 1$, where 1 denotes the identity of $A$.

First, suppose $A$ is finite. Then, by $[14,2.6 .1$, p. 98$], L^{\infty}(\Omega, \mu, A)$ is finite. The conclusion follows directly from Corollary 1 of Proposition 2 in $[5$, p. 256].

Next, suppose $A$ is semi-finite. By [14, p. 157] there exists an increasing net of projections $\left\{e_{\alpha}\right\}$ which are finite and such that sup $e_{\alpha}=1$. Set $A_{\alpha}=L^{\infty}\left(\Omega, \mu, e_{\alpha} A e_{\alpha}\right)$. Then $A_{\alpha}$ is a $W^{*}$-subalgebra of $L^{\infty}(\Omega, \mu, A)$. Suppose $J$ is a closed two-sided ideal of $L^{\infty}(\Omega, \mu, A)$ such that $J \cap C(\Omega, A)=\{0\}$. Then $J \cap C\left(\Omega, e_{\alpha} A e_{\alpha}\right)=\{0\}$ and therefore $J \cap A_{\alpha}=\{0\}$, since $e_{\alpha} A e_{\alpha}$ is a finite factor and the above applies. Now 
let $x \in J^{+}$and set $E_{\alpha}(t)=e_{\alpha}$ for all $t \in \Omega$. It follows that $E_{\alpha} x E_{\alpha} \in J^{+} \cap$ $A_{\alpha}$ and consequently $E_{\alpha} x E_{\alpha}=0$. Since $\left\{E_{\alpha}\right\}$ converges to the identity of $L^{\infty}(\Omega, \mu, A)$ in the $s\left(L^{\infty}(\Omega, \mu, A), L^{1}\left(\Omega, \mu, A_{*}\right)\right)$ topology [14, 1.13.4, p. 30 ] and multiplication is jointly $s\left(L^{\infty}(\Omega, \mu, A), L^{\prime}(\Omega, \mu, A *)\right)$ continuous on uniformly bounded spheres $[14,1.8 .12$, p. 21$]$, it follows that $E_{\alpha} x E_{\alpha}$, converges to $x$. Hence $x=0$. Since $x$ was chosen arbitrarily, $J=\{0\}$.

Finally, suppose $A$ is purely infinite. Since $A *$ is separable, $A$ is countably decomposable $[14,2.1 .9$, p. 80]. Moreover, since the support of $\mu$ is $\Omega$, it follows from [7, Proposition 7, p. 161] that $C(\Omega)$ is countably decomposable. Hence $L^{\infty}(\Omega, \mu, A)$ is a countably decomposable type III (purely infinite) $W^{*}$-algebra [14, 2.6.6, p. 101]. Now, if $J$ is a closed two-sided ideal of $L^{\infty}(\Omega, \mu, A)$ such that $J \cap C(\Omega, A)=$ $\{0\}$, then it follows directly from $[14,4.1 .5$, p. 155] that $J=\{0\}$.

Since $A$ must be either finite, semi-finite or purely infinite, our proof is complete.

3.7. COROllary. Let $\Omega$ be a hyper-Stonean space, $\mu$ a positive normal measure with support $\Omega$, and $A$ a factor with separable predual $A_{*}$. If $J$ is a closed two-sided ideal of $L^{\infty}(\Omega, \mu, A)$ such that $J \cap$ $C(\Omega, A)=\{0\}$, then $J=\{0\}$.

3.8. Theorem. Let $Z$ be a commutative $W^{*}$-algebra and $A$ a factor with separable predual $A_{*}$. If $J$ is an essential ideal of $Z \bar{\otimes} A$, then $J \cap\left(Z \bigotimes_{\alpha_{0}} A\right)$ is an essential ideal of $Z \bigotimes_{\alpha_{0}} A$.

Proof. Just as in 3.6, we may assume $Z \bar{\otimes} A=L^{\infty}(\Omega, \mu, A)$, where $\Omega$ is hyper-Stonean and $\mu$ is a positive normal measure with support $\Omega$. Moreover, we may assume $Z \bigotimes_{\alpha_{0}} A=C(\Omega, A)$. Now suppose $J$ is an essential ideal of $L^{\infty}(\Omega, \mu, A)$ such that $J_{1} \equiv C(\Omega, A) \cap J$ is not essential in $C(\Omega, A)$.

First, we will show that there exists an open and closed subset $G$ of $\Omega$ such that $x(t)=0$ for each $x \in J_{1}$ and $t \in G$. Since $J_{1}^{0} \neq\{0\}$, we may choose a nonzero $y \in J_{1}^{0}$. Because $t \rightarrow\|y(t)\|$ is a continuous map, it is clear that there is an open and closed set $G$ for which $\|y(t)\|>0$ for each $t \in G$. Now suppose there is a $t_{0}$ in $G$ and an $x$ in $J_{1}$ such that $x\left(t_{0}\right) \neq 0$. Then $K_{t_{0}}=\left\{x\left(t_{0}\right): x \in J_{1}\right\}$ is a nonzero closed two-sided ideal of $A$ and moreover, by $3.4, K_{t_{0}}$ is essential in $A$. Since $y \in J_{1}^{0}$, $y\left(t_{0}\right) x\left(t_{0}\right)=0$ for each $x \in J_{1}$. Thus, $y\left(t_{0}\right)=0$ since $K_{t_{0}}$ is essential in A. But this is a contradiction because $y\left(t_{0}\right) \neq 0$. So, $x(t)=0$ for each $x \in J_{1}$ and $t \in G$.

Due to the fact that $J$ is essential in $L^{\infty}(\Omega, \mu, A)$, it is straightforward to show that $\chi_{G} J$ is a nonzero ideal of $L^{\infty}(\Omega, \mu, A)$. Thus, by 3.7, 
$\chi_{G} J \cap C(\Omega, A) \neq\{0\} . \quad$ It follows that there must be an $x \in J_{1}$ for which $x(t) \neq 0$ for some $t \in G$. But this contradicts the defining properties of $G$. Consequently, $J_{1}$ must be essential in $C(\Omega, A)$ and our proof is complete.

3.9. Corollary. Let $\Omega, \mu$, and $A$ be defined as in 3.7. If $J$ is an essential ideal of $L^{\infty}(\Omega, \mu, A)$, then $J \cap C(\Omega, A)$ is an essential ideal of $C(\Omega, A)$.

3.10. Proposition. Let $\Omega, \mu$, and $A$ be defined as in 3.7. If $K$ is an essential closed two-sided ideal in $A$, then $L^{\infty}(\Omega, \mu, K)$ is an essential closed two-sided ideal of $L^{\times}(\Omega, \mu, A)$.

Proof. Let $J=L^{\infty}(\Omega, \mu, K)$ and suppose $J^{0} \neq\{0\}$. Since $J^{0}$ is a closed two-sided ideal, there exists by 3.6 a nonzero $x$ in $J^{0} \cap$ $C(\Omega, A)$. In particular, we have $x y=0$ for all $y \in C(\Omega, K)$. Since $C(\Omega, K)$ is essential in $C(\Omega, A)$ it follows that $x=0$, contradicting that $x \neq 0$. Thus $J$ is essential and our proof is complete.

Let $H$ be a separable Hilbert space, $B(H)$ the bounded linear operators on $H, B_{0}(H)$ the compact operators, and $T(H)$ the trace class operators. It is well known that the dual of $B_{0}(H)$ is $T(H)[14,1.19 .1$, p. 47] and that the predual of $B(H)$ is $T(H)[14,1.15 .3$, p. 39]. Furthermore, $T(H)$ is separable whenever $H$ is separable [14, 2.1.10, p. 81]. These facts will be used in the following examples.

3.11. Example. Let $\Omega$ and $\mu$ be defined as in 3.7 and $H$ as above. Then $L^{\times}\left(\Omega, \mu, B_{0}(H)\right)$ is an essential ideal of $L^{\infty}(\Omega, \mu, B(H))$.

3.12. ExAmple. Let $J$ be a closed two-sided ideal of $L^{\times}(\Omega, \mu, B(H))$. Then $J$ is essential if and only if there exists a closed nowhere dense, possibly empty, subset $E$ of $\Omega$ with the property that for each $x \in J$ and $\epsilon>0$, there is an open neighborhood $V$ of $E$ such that $\|x \mid V\| \leqq \epsilon$. We will denote by $J_{E}$ the essential ideal consisting of all those elements of $L^{x}\left(\Omega, \mu, B_{0}(H)\right)$ which satisfy this property. If $B(H)$ is the complex number system, this essential ideal of $L^{\infty}(\Omega, \mu)$ will be denoted by $I_{E}$.

4. The $\bar{\beta}$ topology. In this section, $A$ will always denote a $W^{*}$-algebra and $A$ * its predual. Let $J$ be an essential ideal of $A$. Recall that the $\beta_{J}$ topology for $A$ is the locally convex topology generated by the family of seminorms $\left\{\lambda_{a}, \rho_{a}\right\}_{a \in J}$, where $\lambda_{a}(x)=\|a x\|$ and $\rho_{a}(x)=\|x a\|$ for all $x \in A$, and the $\bar{\beta}$ topology for $A$ is the inductive limit [13, p. 79] of all the $\beta_{J}$ topologies. As before, let $\mathscr{E}_{A}$, or $\mathscr{E}$ if $A$ is 
understood, denote the family of all essential ideals of $A$. In this section we study the algebra $A$ under the $\bar{\beta}$ topology.

The proofs of 4.1 through 4.5 are by virtue of [20, Corollary $2.7, \mathrm{p}$. 638], simple adaptations of arguments given by Sentilles. Consequently, we do not include them, but rather refer the reader to [18, pp. $317-318]$ and [20, pp. 636-638].

4.1. THEOREM. Let $W$ be a convex, balanced and absorbing subset of $A$. Then $W$ is a $\bar{\beta}$ neighborhood of zero if and only if, for each $r>0$ and $J \in \mathscr{E}$, there is a $\beta_{J}$ neighborhood of zero $V_{J}$ such that $V_{J} \cap\{x:\|x\| \leqq r\} \subseteq W$. Consequently, the strongest locally convex topology for $A$ that agrees with the $\bar{\beta}$ topology on uniformly bounded subsets of $A$ is the $\bar{\beta}$ topology.

4.2. Corollary. The continuity of linear maps on $A_{\bar{\beta}}$ is determined on the uniformly bounded subsets of $A$.

4.3. Corollary. Let $B$ be a locally convex space and $T: A \rightarrow B$ a linear or conjugate linear map. Then $T$ is $\bar{\beta}$ continuous if and only if $T$ is $\beta_{J}$ continuous for each $J \in \mathscr{E}$.

4.4. Corollary. The mappings $x \rightarrow a x, x \rightarrow x a$ and $x \rightarrow x^{*}$ are $\bar{\beta}$ continuous for $x, a \in A$.

4.5. Proposition. The following statements are true: (1) as subsets of $A^{*}, A \frac{6}{\beta}=\cap_{J \in \mathscr{E}} A_{\beta}^{*}$; (2) if, for each $J \in \mathscr{E}, \beta_{J}$ is the Mackey topology of the dual pair $\left\langle A, A_{\beta}^{*}\right\rangle$, then $\bar{\beta}$ is the Mackey topology of the dual pair $\langle A, A \underset{\beta}{*}\rangle$.

Note that $A_{*}$ is a uniformly closed subspace of $A^{*}$.

4.6. THEOREM. For the dual pair $\langle A, A *\rangle$, we have $\tau(A, A *) \leqq \bar{\beta}$, where $\tau\left(A, A_{*}\right)$ denotes the Mackey topology of the dual pair $\langle A, A *\rangle$.

Proof. By virtue of $[14,1.16 .7$, p. 41$], A$ can be viewed as a weakly closed self-adjoint subalgebra of $B(H)$, where $H$ is some Hilbert space with the property $H=\{T(h): T \in A, h \in H\}$. Let $J$ be an essential ideal in $A$. By the Cohen-Hewitt factorization theorem [10, Theorem 2.5, p. 151], $H_{0} \equiv\{T(h): T \in J, h \in H\}$ is a closed subspace of $H$. Furthermore, since $J$ is essential, $H=H_{0}$. It follows that the $\beta_{J}$ topology is stronger than the strong operator topology and therefore stronger than the $s\left(A, A_{*}\right)$ topology on uniformly bounded spheres [14, 1.15.2, p. 35]. Moreover, due to the fact that the map 
$x \rightarrow x^{*}$ is $\beta_{J}$ continuous and multiplication is jointly $\beta_{J}$ continuous on uniformly bounded spheres, the $\beta_{J}$ topology is stronger than the $s^{*}\left(A, A_{*}\right)$ topology on uniformly bounded spheres. But, Akemann has shown that on uniformly bounded spheres the $\tau(A, A *)$ and $s^{*}(A, A *)$ topologies agree [1, Theorem II.7, p. 292]. The conclusion now follows from 4.1.

4.7. Corollary. The Banach space $A *$ is equal to $A \frac{*}{\beta}$ if and only if $\bar{\beta}=\tau\left(A, A_{*}\right)$.

4.8. Corollary. A set $V \subseteq A$ is $\bar{\beta}$ bounded if and only if $V$ is uniformly bounded.

4.9. Corollary. The unit ball of $A$ is closed in the $\bar{\beta}$ topology.

4.10. Proposition. The following statements are equivalent

(1) $\mathscr{E}=\{A\}$

(2) $\bar{\beta}$ is normable

(3) $\bar{\beta}$ is metrizable

(4) $\bar{\beta}$ is bornological

(5) $\bar{\beta}$ is barrelled.

Proof. It is clear that (1) implies (2), (2) implies (3), and (3) implies (4). Assume (4) holds, that is, $\bar{\beta}$ is bornological. Then $\bar{\beta}$ is the strongest locally convex topology on $A$ with the same class of bounded sets. Thus, by $4.8 \bar{\beta}$ is the norm topology and therefore barrelled. Now assume $\bar{\beta}$ is barrelled. It follows that the unit ball $B_{1}$ of $A$ is a $\bar{\beta}$ neighborhood of zero. Thus the norm and $\beta_{J}$ topologies agree on $A$ for all $J \in \mathscr{E}$. From [8, 3.2.4, p. 78] we have, for $J \in \mathscr{E}$, $A=M(J)=J$ and our proof is complete.

4.11. Proposition. Suppose $\left\{A_{\alpha}\right\}$ is a family of $W^{*}$-algebras such that $A=\Sigma_{\alpha \in \pi} \bigoplus A_{\alpha}$. Then $A \frac{*}{\beta}=\left(\sum_{\alpha \in \pi} \oplus\left(A_{\alpha}\right)_{\beta}^{*}\right)_{l,}[14,1.1 .5$, p. 2]. Consequently, $A_{\beta}^{*}=A *$ if and only if $\left(A_{\alpha}\right)_{\beta}^{*}=\left(A_{\alpha}\right) *$ for each $\alpha \in \pi$.

Proof. Note that essential ideals of $A$ of the form $\left(\Sigma_{\alpha \in \pi} \oplus J_{\alpha}\right)_{0}$, where $J_{\alpha}$ is essential in $A_{\alpha}$, generate the $\bar{\beta}$ topology for $A$. By $\left(\Sigma_{\alpha \in \pi} \oplus J_{\alpha}\right)_{0}$ we mean those $\left\{x_{\alpha}\right\}$ in $A$ such that $x_{\alpha} \in J_{\alpha}$ and $\alpha \rightarrow\left\|x_{\alpha}\right\|$ vanishes at infinity. By using this fact together with 4.3 and 4.13 , the proof becomes straightforward.

4.12. Proposition. Let $f$ be a hermitian $\bar{\beta}$ continuous linear functional on $A$. Then there exists a unique decomposition $f=f_{1}-f_{2}$, where $f_{1}$ and $f_{2}$ are positive $\bar{\beta}$ continuous linear functionals such that $\|f\|=\left\|f^{+}\right\|+\left\|f^{-}\right\|$. 
Proof. The proof follows directly from [6, 12.3.4, p. 245], [21, Corollary 2.6, p. 164] and 4.3.

4.13. COROllary. The space $A \frac{*}{\beta}$ is the linear span of its positive elements.

For $f \in A^{*}$ and $x, y \in A$ we define the elements of $A^{*} x \cdot f, f \cdot x$ and $x \cdot f \cdot y$ by $(x \cdot f)(a)=f(a x),(f \cdot x)(a)=f(x a)$ and $(x \cdot f \cdot y)(a)$ $=f($ yax $)$ for all $a \in A$.

4.14. Proposition. Suppose $J$ is an essential ideal of $A$. Then $A \frac{*}{B}$ is the linear span of all linear functionals in $A \underset{B}{*}$ of the form $x \cdot g \cdot x$, where $x \in J^{+}$and $g$ is a positive $\bar{\beta}$ continuous linear functional on $A$.

Proof. Let $f \in A_{B}^{*}$. Suppose $\left\{e_{\lambda}\right\}$ is a positive approximate identity for $J$. Since $f$ is also $\beta_{J}$ continuous, it follows from [20, Corollary 2.2, p. 635] that $\lim e_{\lambda} \cdot f=\lim f \cdot e_{\lambda}=f$. Due to the fact that $A * \frac{*}{\beta}$ is both a left and right $J$-module, we see that $f=a \cdot h \cdot b$ by virtue of [19, Theorem 2.1, p. 142], where $h \in A_{\beta}^{*}$. By a variant of [19, Theorem 2.1, p. 142] there exist elements $x, y, z$ in $J$ such that $x \geqq 0$ and $a=x y$ and $b=z x$. Thus $f=x \cdot g \cdot x$, where $g=y \cdot h \cdot z$. The remainder of the proof follows immediately from 4.13.

4.15. Proposition. Suppose $A$ is a factor and $J$ is the smallest closed two-sided ideal of $A$. Then $A \underset{B}{*}=A_{\beta}^{*}$.

Proof. The proof is trivial.

4.16. Proposition. Suppose $A$ is a factor. Then $A \frac{*}{B}=A *$ if and only if $A$ is of type $I$.

Proof. If $A$ is a type I factor, then $A=B(H)$ for some Hilbert space $H$. For this case $A_{*}=T(H)$, the trace class operators, and $A_{B}^{*}=A_{\beta}^{*}$, where $J=B_{0}(H)$. But $T(H)=B_{0}(H)^{*}=A_{\beta}^{*},[20$, Corollary 2.3 , p. 635]. So, the first part of our proof is complete.

Now suppose $A \underset{B}{*}=A_{*}$. By 4.15 and [20, Corollary 2.3, p. 635], $A_{\beta}^{*}=A_{\beta_{J}}^{*}=J^{*}=A *$. So $A_{\beta_{J}}^{* *}=A$ and by [23, Theorem 5.1, p. 533], $A$ is of type $\mathrm{I}$.

5. The main results. In this section, $A$ will denote a $W^{*}$-algebra, $A *$ its unique predual, $\mathscr{E}_{A}$ the essential ideals of $A$, or $\mathscr{E}$ if $A$ is understood, and $A \frac{*}{\beta}$ the dual of $A$ under the $\bar{\beta}$ topology. We will now state one of the main results of the section. 
THEOREM I. If $A$ is a countably decomposable type I $W^{*}$-algebra, then $A_{\beta}^{*}=A *$. Consequently, the $\bar{\beta}$ topology is the Mackey topology of the dual pair $\langle A, A *\rangle$.

Before we proceed with the proof of Theorem I, we will need the following three lemmas.

5.1. Lemma. If $A$ is a countably decomposable type $I_{n} W^{*}$ algebra ( $n$ a cardinal number), then $n \leqq \aleph_{0}$. Consequently, $A$ is *isomorphic to $\Sigma_{\alpha \in \Gamma} \oplus L^{\infty}\left(\Omega_{\alpha}, \mu_{\alpha}, B(H)\right)$, where $\Omega_{\alpha}$ is hyper-Stonean, $\mu_{\alpha}$ is a finite positive normal measure with support $\Omega_{\alpha}$, and the dimension of the Hilbert space $H$ is $n$.

Proof. The proof follows from [14, 2.3.3, p. 89] and 3.5.

In the next two lemmas we will assume $\Omega$ is hyper-Stonean, $\mu$ is a finite positive normal measure with support $\Omega$, and $H$ is a separable Hilbert space.

5.2. LEMMA. Let $B_{1}$ be the set of all finitely-valued functions $x$ in $C\left(\Omega, B_{0}(H)\right)^{+}$with $\|x\| \leqq 1$. Then $\bigcup_{J \in \mathscr{Q}} C l_{\beta J}\left(B_{1}\right)$ is equal to $D_{1}$, the set of all $x$ in $L^{\infty}\left(\Omega, \mu, B_{0}(H)\right)^{+}$with $\|x\| \leqq 1$. Here, $C l_{\beta_{J}}\left(B_{1}\right)$ denotes the closure of $B_{1}$ in the $\beta_{J}$ topology.

Proof. By 4.9, $\cup_{J \in \mathscr{\ell}} C l_{\beta_{J}}\left(B_{1}\right) \subseteq D_{1}$. Let $x \in D_{1}$. By [11, Corollary 1 and Corollary 2, p. 73], there exists a sequence $\left\{x_{n}\right\}_{n=1}^{\infty}$ in $D_{1}$ of countably-valued functions such that $\left\|x_{n}-x\right\| \rightarrow 0$. Thus, it suffices to assume that $x$ is countably-valued. Let $\left\{T_{i}\right\}_{i=1}^{\infty}$ be the values in $B_{0}(H)^{+}$ assumed by $x$. Then set $E_{i}=\left\{t: x(t)=T_{i}\right\}$. By virtue of 2.2, 2.5 and 2.7, we may assume $x=\sum_{i=1}^{\infty} T_{i} \chi_{E_{t}}$, where $E_{i} \cap E_{j}=\varnothing, i \neq j$, and $E_{i}$ is open and closed. Let $E=\cup_{i=1}^{\infty} E_{i} \backslash \cup_{i=1}^{\infty} E_{i}$ and suppose $E \neq \varnothing$. Then $E$ is closed and, by 2.5 and 2.7 , nowhere dense. Let $J_{E}$ be the ideal of $L^{\propto}\left(\Omega, \mu, B_{0}(H)\right)$ defined in 3.12. We shall show that $x_{n} \rightarrow x$ in the $\beta_{J_{E}}$ topology where $x_{n}=\sum_{i=1}^{n} T_{i} \chi_{E_{i}}$. Given $z \in J_{E}$ and $\epsilon>0$, there exists an open set $V_{\epsilon} \supseteq E$ such that $\|z(t)\|<\epsilon / 2\|x\|$ for almost all $t \in V_{\epsilon}$. Since $x$ is continuous on $\tilde{V}_{\epsilon}$, the complement of $V_{\epsilon}$, the functions $t \rightarrow \| x_{n}(t)-$ $x(t) \|$ form a decreasing sequence of positive continuous functions that converge pointwise to 0 on the compact set $\tilde{V}_{\epsilon}$. Consequently, by Dini's Theorem there exists an integer $N$ such that $\left\|x_{n}(t)-x(t)\right\|<$ $\epsilon /\|z\|$ for all $t \in \tilde{V}_{\epsilon}$ and $n \geqq N$. It easily follows that $\left\|z x_{n}-z x\right\|<\epsilon$ and $\left\|x_{n} z-x z\right\|<\epsilon$ for $n \geqq N$. Thus, $x_{n} \rightarrow x$ in the $\beta_{J_{E}}$ topology. If $E=\varnothing$, then by Dini's theorem $x_{n} \rightarrow x$ uniformly on $\Omega$. Therefore $x \in \cup_{J \in \mathscr{G}} C l_{\beta_{J}}\left(B_{1}\right)$ and our proof is complete.

In the following lemma let $A$ denote the $W^{*}$-algebra $L^{\infty}(\Omega, \mu, B(H))$. 
5.3. Lemma. If $F \in A$ * and $x$ is a finitely valued function in $C\left(\Omega, B_{0}(H)\right)$, then $x \cdot F \in A *$.

Proof. It is straightforward to verify, by utilizing the spectral theorem for compact operators, 4.13 , and 2.5 , that we can make the following assumptions: (1) $x=\chi_{G} P$, where $G$ is an open and closed subset of $\Omega$ and $P$ is a one-dimensional projection on $H$; (2) $F$ is positive. First, we will show that $x \cdot F \cdot x \in A *$, or equivalently, $x \cdot F \cdot x$ is normal $\left[14,1.13 .2\right.$, p. 28]. Let $\left\{z_{\alpha}\right\}$ be an increasing net in $A^{+}$ with $z=\sup z_{\alpha}$. Since $x \cdot F \cdot x\left(z_{\alpha}\right)=F\left(x z_{\alpha} x\right)$ and $F$ is $\bar{\beta}$ continuous, it will suffice to show $x z_{\alpha} x \rightarrow x z x$ in the $\bar{\beta}$ topology.

Let $E \subseteq \Omega$ be a closed nowhere dense set, $I_{E}$ the ideal of $L^{\infty}(\Omega, \mu)$ as defined in 3.12 and $J_{E}$ the corresponding ideal of $L^{\infty}\left(\Omega, \mu, B_{0}(H)\right.$ ) (see 3.12). For $y \in J_{E}$ we have

$$
\begin{aligned}
& \left\|y(t) x(t)\left[z(t)-z_{\alpha}(t)\right] x(t)\right\| \\
& \quad \leqq \sup \left\{\left\|y(t) x(t)\left[z(t)-z_{\alpha}(t)\right] x(t) h\right\|: h \in H,\|h\| \leqq 1\right\} \\
& \quad \leqq \sup \left\{\left|\left\langle h, h_{0}\right\rangle\left\langle\left(z(t)-z_{\alpha}(t)\right)\left(h_{0}\right), h_{0}\right\rangle\right|\left\|y(t)\left(h_{0}\right)\right\|: h \in H,\|h\| \leqq 1\right\} \\
& \quad \leqq\left|\left\langle\left(z(t)-z_{\alpha}(t)\right)\left(h_{0}\right), h_{0}\right\rangle\right|\|y(t)\|,
\end{aligned}
$$

where $P\left(h_{0}\right)=h_{0}$ and $\left\|h_{0}\right\|=1 . \quad$ By [11, Theorem 2.8.5, p. 34] and [11, Theorem 3.5.2, p. 72], the map $t \rightarrow\|y(t)\|$ is measurable and thus equal to, almost everywhere, a continuous function that vanishes on $E$. Therefore, it sufficies to find a closed nowhere dense set $E$ for which $\phi_{\alpha}(t) \equiv\left\langle z_{\alpha}(t)\left(h_{0}\right), h_{0}\right\rangle$ converges to $\phi(t) \equiv\left\langle z(t)\left(h_{0}\right), h_{0}\right\rangle$ in the $\beta_{I_{E}}$ topology, for then $x z_{\alpha} x$ will converge to $x z x$ in the $\beta_{J_{E}}$ topology, and hence, in the $\bar{\beta}$ topology.

Since $x z_{\alpha} x \rightarrow x z x$ in the $\sigma\left(A, A_{*}\right)$ topology $[14,1.7 .4, \mathrm{p} .15]$ and the predual of $L^{\infty}(\Omega, \mu, B(H))$ is $L^{1}(\Omega, \mu, T(H))$, it is easy to show that $\int_{\Omega} \phi_{\alpha}(t) d \mu \rightarrow \int_{\Omega} \phi(t) d \mu$. By virtue of 2.4 we can choose functions $f_{\alpha}, f$ in $C(\Omega)$ such that $f_{\alpha}=\phi_{\alpha}$ and $f=\phi$ almost everywhere. Note that $f_{\alpha}$ is an increasing net with $f \geqq f_{\alpha}$, so $f \geqq \sup f_{\alpha} \equiv f^{\prime}$. Since $\mu$ is a positive normal measure, $\int_{\Omega} f_{\alpha} d \mu \rightarrow \int_{\Omega} f^{\prime} d \mu$. Hence, $\int_{\Omega}\left(f-f^{\prime}\right) d \mu=0$, or equivalently, $f=f^{\prime}$. Now, let $E$ be the closure of $\left\{t: f(t)>\sup f_{\alpha}(t)\right\}$. By 2.1, 2.2, 2.5 and 2.7, $E$ is nowhere dense. By virtue of Dini's theorem, it is straightforward to show that $f_{\alpha} \rightarrow f$ in the $\beta_{I_{E}}$ topology for $C(\Omega)$ and consequently $x z_{\alpha} x \rightarrow x z x$ in the $\beta_{J_{E}}$ topology for $L^{\infty}(\Omega, \mu, B(H))$. Thus $x \cdot F \cdot x \in A *$.

Finally, we must show $x \cdot F \in A *$ Suppose $x_{\alpha} \rightarrow 0$ for the 
$s^{*}\left(A, A_{*}\right)$ topology where $\left\|x_{\alpha}\right\| \leqq 1$. Then $x_{\alpha}^{*} \rightarrow 0$ for the $s^{*}\left(A, A_{*}\right)$ topology and by $[14,1.8 .9$, p. 20$]$ and $[14,1.8 .12$, p. 21$]$ it follows that $x_{\alpha}^{*} x_{\alpha} \rightarrow 0$ for the $s\left(A, A_{*}\right)$ topology. By utilizing the Schwartz inequality $[14$, p. 9], $[14,1.8 .10$, p. 21] and the above result for $x \cdot F \cdot x$ it is easy to show that $(x \cdot F)\left(x_{\alpha}\right) \rightarrow 0$. Thus $x \cdot F \in A *$ and our proof is complete.

Proof of Theorem I. By [14, 2.3.2, p. 89], $A=\Sigma_{n \in \Gamma} \oplus A_{n}$, where each $A_{n}$ is a type $\mathrm{I}_{n} W^{*}$-algebra. By virtue of 4.11 we may assume $A$ is of type $I_{n}$ and consequently by 5.1 and 4.11 we may assume $A=L^{\infty}(\Omega, \mu, B(H))$ where $\Omega$ is hyper-Stonean, $\mu$ is a positive normal measure with support $\Omega$, and $H$ is a separable Hilbert space.

Let $F \in L^{\infty}(\Omega, \mu, B(H))$ * such that $F \geqq 0$. By 4.14 and 3.11 we may assume $F=x \cdot G \cdot x$ for some $x \in L^{\infty}\left(\Omega, \mu, B_{0}(H)\right)^{+}$and $G$ a positive $\bar{\beta}$ continuous linear functional on $L^{\infty}(\Omega, \mu, B(H))$. Clearly, we may assume $\|x\| \leqq 1$. By 5.2 there exists an essential ideal $J$ in $L^{\infty}(\Omega, \mu, B(H))$ such that $x \in C_{\beta_{J}}\left(B_{1}\right)$. Consequently, there exists a net $\left\{x_{\alpha}\right\}$ in $B_{1}$ that converges to $x$ in the $\beta_{J}$ topology. By 4.14 we may assume $G=y \cdot G_{1} \cdot y$ for some $y \in J^{+}$and $G_{1}$ a positive $\bar{\beta}$ continuous linear functional on $L^{\infty}(\Omega, \mu, B(H))$. Therefore $x_{\alpha} \cdot G \cdot x_{\alpha} \rightarrow F$ uniformly. Hence, by virtue of 5.3, $F \in A_{*}$. Since $A *$ is the linear span of its positive linear functionals, $A_{\beta}^{*}=A_{*}$. That $\bar{\beta}$ is the Mackey topology of the dual pair $\left\langle A, A_{*}\right\rangle$, is an immediate consequence of 4.7 and our proof is now complete.

For a type I $W^{*}$-algebra, the condition of being countably decomposable is not necessary for Theorem I to hold. In fact, Theorem I holds for $A=B(H), H$ not separable (see 4.16), and for any commutative $W^{*}$-algebra $Z$. Moreover, it is easy to see from our proof, that Theorem I holds for $Z \bar{\otimes} B(H)$ where $H$ is a separable Hilbert space. It is of interest to note that for the $W^{*}$-algebra $L^{\infty}=L^{\infty}(X, \nu)$, where $X$ completely regular and $\nu$ is a compact regular Borel measure on $X$, we have $\bar{\beta}=\tau\left(L^{\infty}, L^{1}\right)$ where $L^{1}=L^{1}(X, \nu)$. Thus, $\bar{\beta}$ is the mixed topology considered by Dazord and Jourlin [4]. These results lead us to the following two related questions.

5.4. Question. Suppose $A$ is a type $I W^{*}$-algebra that is not countably decomposable. Must $A \underset{\beta}{*}$ be equal to $A *$ ?

5.5. Question. Let $A$ be a countably decomposable $W^{*}$-algebra such that $A_{\beta}^{*}=A_{*}$. Must $A$ be a type I $W^{*}$-algebra? In other words, does the converse of Theorem I hold? 
Our next result suggests that the converse of Theorem I may indeed be true. We will now view $A$ as a $W^{*}$-algebra on a separable Hilbert space.

Theorem II. Let $A=\int_{\Gamma} A(t) \mu(d t)$ be the direct integral decomposition of $A$ into factors [17, Corollary 10, p. 53]. Let $B$ be a factor and define $\Lambda$ to be the set of all $t \in \Gamma$ for which $A(t)$ is spatially isomorphic to $B$. If $A \underset{\beta}{*}=A_{*}$ and $\mu(\Lambda)>0$, then $B$ must be a type $I$ factor.

Proof. By virtue of [17, Theorem 2, p. 228], 4.11, and 3.5, we need only consider the case $A=L^{\infty}(\Omega, \mu, B)$, where $\Omega$ is hyper-Stonean, $\mu$ is a positive normal measure with support $\Omega$, and $\mu(\Omega)=1$. Now, let $D$ be the set of all elements in $C(\Omega, B)$ of the form $\sum_{k=1}^{n} x_{k} \chi_{E_{k}}$, where $x_{k} \in B$ and $\left\{E_{k}\right\}_{k=1}^{n}$ are pairwise disjoint sets that are both open and closed. Since $D$ is a *-subalgebra of $C(\Omega, B)$ that separates points and contains the identity, we have by $[6,11.5 .3$, p. 234$]$ that $D$ is uniformly dense in $C(\Omega, B)$. Consequently, it follows from [14, 1.22.3, p. 61] and $\left[14\right.$, p. 67] that $D$ is $\sigma\left(A, A_{*}\right)$ dense in $L^{\infty}(\Omega, \mu, B)$. Now, for $f \in\left(B *_{\beta_{B}}\right)^{+}$ define $\tilde{f}$ on $D$ as follows: for $x=\sum_{k=1}^{n} x_{k} \chi_{E_{k}}$, set $\tilde{f}(x)=\sum_{k=1}^{n}$ $f\left(x_{k}\right) \mu\left(E_{k}\right)$. We will now show that $\tilde{f}$ is continuous on the unit ball of $D$ for the relative $\bar{\beta}_{A}$ topology. By 4.1 and 4.3 , it will suffice to show that $\tilde{f}$ is continuous on the unit ball of $D$ in the relative $\beta_{J}$ topology for each $J \in \mathscr{E}_{A}$.

Let $J$ be a closed two-sided essential ideal of $A, I_{0}$ the smallest closed two-sided ideal of $B$, and $\epsilon>0$. Since $f$ is $\bar{\beta}_{B}$ continuous on $B$, there exists a $b \in I_{0}$ such that $|f(x)| \leqq \epsilon / 2$ whenever $\|b x\|+\|x b\| \leqq$ 1. Now set $J_{1}=J \cap C(\Omega, B)$. By 3.9, $J_{1}$ is essential in $C(\Omega, B)$, so the set $E=\left\{t \in \Omega: x(t)=0\right.$ for all $\left.x \in J_{1}\right\}$ is a closed nowhere dense subset of $\Omega$. Since $\mu$ is normal, there exists an open and closed set $G \supseteq E$ such that $\mu(G)<\epsilon / 2\|f\|$. For each $t_{0} \in \tilde{G}$, the complement of $G$, the set $\left\{x\left(t_{0}\right): x \in J_{1}\right\}$ contains $I_{0}$. Therefore, it is straightforward to show that there exists a subset $V_{t_{0}}$ of $\tilde{G}$ and an element $a_{0}$ in $J_{1}$ that satisfy the following:

(1) $t_{0} \in V_{t_{0}}$ and $V_{t_{0}}$ is both open and closed;

(2) $\left\|a_{0}(t)-b\right\|<1 / 8$ for $t \in V_{t_{0}}$ and $a_{0}(t)=0$ otherwise. Since $\tilde{G}$ is compact and $\left\{V_{t}\right\}_{t \in \tilde{G}}$ is an open cover, there exists a finite collection $\left\{V_{t_{i}}\right\}_{i=1}^{m}$ that covers $\tilde{G}$. Due to the fact that each set $V_{t i}$ is both open and closed, we can construct an element $a$ in $J_{1}$ such that $\|a(t)-b\|<1 / 4$ for $t \in \tilde{G}$ and $a(t)=0, t \in G$. Now let $x$ be an element of the unit ball of $D$, where $x=\sum_{k=1}^{n} x_{i} \chi_{E_{i}}$, and suppose $\|x a\|+\|a x\| \leqq 1 / 2$. We may assume that, for some positive integer $k, E_{1}, E_{2}, \cdots, E_{k}$ are subsets of $G$ 
and $E_{k+1}, \cdots, E_{n}$ are subsets of $\tilde{G}$. Since $\|x\| \leqq 1$ and $\|a x\|+\|x a\| \leqq 1 / 2$, it is easy to show that $\left\|x_{i} b\right\|+\left\|b x_{i}\right\| \leqq 1$ for $i=k+1, \cdots, n$. Therefore

$$
\begin{aligned}
|\tilde{f}(x)| & \leqq \sum_{i=1}^{k}\left|f\left(x_{i}\right)\right| \mu\left(E_{i}\right)+\sum_{i=k+1}^{n}\left|f\left(x_{i}\right)\right| \mu\left(E_{i}\right) \\
& \leqq\|f\| \mu(G)+(\epsilon / 2) \mu(\tilde{G})<\epsilon / 2+\epsilon / 2=\epsilon .
\end{aligned}
$$

Thus, $\tilde{f}$ is $\bar{\beta}_{A}$ continuous on the unit ball of $D$. Since $A_{\beta_{A}}^{*}=A *$, the $\bar{\beta}_{A}$ topology is the Mackey topology of the dual pair $\langle A, A *\rangle$. Consequently, it follows from $[14,1.9 .1$, p. 22] and $[14,1.8 .10$, p. 21] that $\tilde{f}$ can be extended uniquely to a $\bar{\beta}_{A}$ continuous positive linear functional on $A$. Hence $\tilde{f} \in A$ * and this implies $f \in B_{*}$. But, by 4.16 this can only happen when $B$ is a type $I$ factor and our proof is complete.

\section{REFERENCES}

1. C. A. Akemann, The dual space of an operator algebra, Trans. Amer. Math. Soc., 126 (1967), 286-302.

2. R. C. Buck, Bounded continuous functions on a locally compact space, Michigan Math. J., 5 (1958), 95-104.

3. R. C. Busby, Double centralizers and extensions of $C^{*}$-algebras, Trans. Amer. Math. Soc., 132 (1968), 79-99.

4. J. Dazord and M. Jourlin, La dualite entre les espaces $L^{\prime}$ and $L^{\infty}$ (submitted).

5. J. Dixmier, Les algebras d'operateurs dans l'espace Hilbertien, 2nd edition, Gauthier-Villars, Paris, 1969.

6. Les $C^{*}$-algebras et leurs representations, 2nd edition, Cahiers Scientifiques, fasc. 29, Gauthier-Villars, Paris, 1969.

7. - Sur certain espaces consideres par M. H. Stone, Summa Brasil. Math., 2 (1951), fasc. $11,151-182$.

8. R. A. Fontenot, Approximate identities and strict topologies, Ph.D. dissertation, Louisiana State University, 1972.

9. L. Gillman and M. Jerison, Rings of continuous functions, Princeton, 1960.

10. E. Hewitt, The ranges of certain convolution operators, Math. Scand., 15 (1964), 147-155.

11. E. Hille and R. S. Phillips, Functional analysis and semi-groups, Amer. Math. Soc. Coll. Publ.

31, Providence, 1957.

12. S. E. Mosiman and R. F. Wheeler, The strict topology in a completely regular setting: relations to topological measure theory, Canad. J. Math., 24 (1972), 873-890.

13. A.'P. Robertson and W. J. Robertson, Topological vector spaces, Cambridge Univ. Press, New York, 1964.

14. S. Sakai, $C^{*}$-algebras and $W^{*}$-algebras, Springer-Verlag, New York, 1971.

15. H. H. Schaefer, Topological vector spaces, Macmillan, New York, 1964.

16. K. S. Scheinberg, Topologies which generate a complete measure algebra, Advances in Math. 7 (1971), 231-239.

17. J. T. Schwartz, $W^{*}$-algebras, Gordon and Breach, New York, 1967.

18. F. D. Sentilles, Bounded continuous functions on a completely regular space, Trans. Amer. Math. Soc., 168 (1972), 311-336.

19. F. D. Sentilles and D. C. Taylor, Factorization in Banach algebras and the general strict topology, Trans. Amer. Math. Soc., 142 (1969), 141-152.

20. D. C. Taylor, The strict topology for double centralizer algebras, Trans. Amer. Math. Soc., 150 (1972), 633-643. 
21. Interpolation in algebras of operator fields, J. Functional Analysis, 10 (1972), 159-190.

22. A general Phillips theorem for $C^{*}$-algebras and some applications, Pacific J. Math., 40 (1972), 477-488.

23. B. Tomiuk and P. K. Wong, The Arens product and duality in $B^{*}$-algebras, Proc. Amer. Math. Soc., 25 (1970), 529-535.

24. R. F. Wheeler, The strict topology, separable measures and paracompactness, Pacific J. Math., (to appear).

25. The strict topology for P-spaces, Proc. Amer. Math. Soc., 41 (1973), $466-472$.

26. W. Wils, Two-sided ideals in $W^{*}$-algebras, J. Reine Angew. Math., 244 (1970), 55-68.

Received March 5, 1974. This research was supported in part by the National Science Foundation, under contract No. GP-38884.

Department of Mathematics

Montana State University 

D. E. Bennett, Strongly unicoherent continua ............................. 1

Walter R. Bloom, Sets of p-spectral synthesis ................................ 7

R. T. Bumby and D. E. Dobbs, Amitsur cohomology of quadratic extensions: Formulas and number-theoretic examples ................. 21

W. W. Comfort, Compactness-like properties for generalized weak topological sums

D. R. Dunninger and J. Locker, Monotone operators and nonlinear biharmonic boundary value problems ...

T. S. Erickson, W. S. Martindale, 3rd and J. M. Osborn, Prime nonassociative algebras

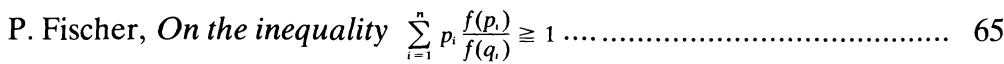

G. Fox and P. Morales, Compact subsets of a Tychonoff set ............... 75

R. Gilmer and J. F. Hoffmann, A characterization of Prüfer domains in terms of polynomials ......................................................... 81

L. C. Glaser, On tame Cantor sets in spheres having the same projection in each direction ......................................................... 87

Z. Goseki, On semigroups in which $X=X Y X=X Z X$ if and only if

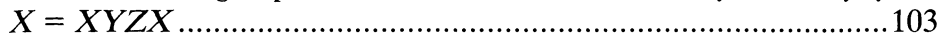

E. Grosswald, Rational valued series of exponentials and divisor

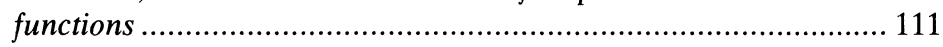

D. Handelman, Strongly semiprime rings ...................................... 115

J. N. Henry and D. C. Taylor, The $\bar{\beta}$ topology for $w^{*}$-algebras ............. 123

M. J. Hodel, Enumeration of weighted p-line arrays .......................... 141

S. K. Jain and S. Singh, Rings with quasiprojective left ideals .............. 169

S. Jeyaratnam, The diophantine equation $Y(Y+m)(Y+2 m) \times$

$$
(Y+3 m)=2 X(X+m)(X+2 m)(X+3 m) \ldots \ldots \ldots \ldots \ldots \ldots \ldots . . .183
$$

$\mathrm{R}$. Kane, On loop spaces without $p$ torsion .........................................189

Alvin J. Kay, Nonlinear integral equations and product integrals ..........203

A. S. Kechris, Countable ordinals and the analytic hierarchy, I ...........223

Ka-Sing Lau, A representation theorem for isometries of $C(X, E) \ldots \ldots . .229$

I. Madsen, On the action of the Dyer-Lashof algebra in $H_{*}(G)$..........235

R. C. Metzler, Positive linear functions, integration, and Choquet's theorem ........................................................................................ 277

A. Nobile, Some properties of the Nash blowing-up ............................297

G. E. Petersen and G. V. Welland, Plessner's theorem for Riesz conjugates 


\section{Pacific Journal of Mathematics}

\section{Vol. 60, No. $1 \quad$ September, 1975}

Donald Earl Bennett, Strongly unicoherent continua ................ 1

Walter Russell Bloom, Sets of p-spectral synthesis ................ 7

Richard Thomas Bumby and David Earl Dobbs, Amitsur cohomology of

quadratic extensions: formulas and number-theoretic examples .......

W. Wistar (William) Comfort, Compactness-like properties for generalized

weak topological sums ...................................

Dennis Robert Dunninger and John Stewart Locker, Monotone operators

and nonlinear biharmonic boundary value problems ..............

Theodore Erickson, Wallace Smith Martindale, III and J. Marshall Osborn,

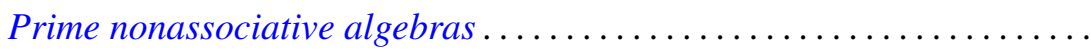

Pál Fischer, On the inequality $\sum_{i=0}^{n}\left[f\left(p_{i}\right) / f\left(q_{i}\right)\right] p_{i} \geq i \ldots \ldots \ldots \ldots \ldots$

Geoffrey Fox and Pedro Morales, Compact subsets of a Tychonoff set.......

Robert William Gilmer, Jr. and Joseph F. Hoffmann, A characterization of

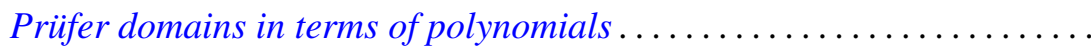

Leslie C. Glaser, On tame Cantor sets in spheres having the same projection

in each direction . ...................................

Zensiro Goseki, On semigroups in which $x=x y x=x z x$ if and only if

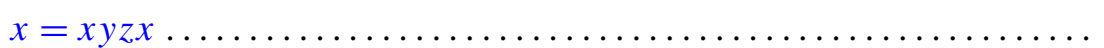

Emil Grosswald, Rational valued series of exponentials and divisor

functions.

David E. Handelman, Strongly semiprime rings

Jackson Neal Henry and Donald Curtis Taylor, The $\bar{\beta}$ topology for

$W^{*}$-algebras

Margaret Jones Hodel, Enumeration of weighted p-line arrays ...

Surender Kumar Jain and Surjeet Singh, Rings with quasi-projective left

ideals.

S. Jeyaratnam, The Diophantine equation

$$
Y(Y+m)(Y+2 m)(Y+3 m)=2 X(X+m)(X+2 m)(X+3 m) \ldots
$$

Richard Michael Kane, On loop spaces without $p$ torsion

Alvin John Kay, Nonlinear integral equations and product integrals ...

Alexander S. Kechris, Countable ordinals and the analytical hierarchy.

$I$.

Ka-Sing Lau, A representation theorem for isometries of $C(X, E)$

Ib Henning Madsen, On the action of the Dyer-Lashof algebra in $H_{*}(G)$

Richard C. Metzler, Positive linear functions, integration, and Choquet's

theorem.

Augusto Nobile, Some properties of the Nash blowing-up

Gerald E. Peterson and Grant Welland, Plessner's theorem for Riesz. 